SSN 2519-7398 (Versión electrónica)

DOI: http://dx.doi.org/10.21704/ac.v78i2.1053

(C) Universidad Nacional Agraria La Molina, Lima - Perú

\title{
Uso del modelo "Brazilian Regional Atmospheric Modeling System” (brams) en el análisis del huaico de Chosica en Lima Perú
}

\author{
Using the model "Brazilian Regional Atmospheric Modeling System" (brams) in the analysis of \\ Chosica mudslide in Lima Perú
}

\author{
Jerónimo García Villanueva ${ }^{*}$; Bremilda Andrea Sutizal sánchez ${ }^{2}$ \\ *Autor de correspondencia
}

\begin{abstract}
Resumen
En la actualidad existen modelos numéricos que permiten disponer de informaciones para las investigaciones y análisis en lugares con escasez de datos meteorológicos observados. El modelo regional BRAMS cuenta con datos de pronóstico, procedentes del modelo global del CPTEC, que deberían usarse para el proceso de downscaling dinámico cuyos resultados son útiles en el análisis de las condiciones del tiempo pronosticado en zonas de eventos climáticos de alto riesgo para prevenir sus efectos negativos y aprovechar la capacidad de predicción del modelo. En este trabajo se usó el modelo BRAMS para determinar el origen de las precipitaciones que ocasionaron el huaico en Chosica en marzo del 2015. Se determinó la distribución altitudinal de precipitación pronosticada con cinco días de anticipación y con precipitaciones observadas; también se analizaron los vientos en superficie y altura para identificar los flujos asociados (los sistemas del Pacífico o los sistemas del Este) con las precipitaciones; asimismo se analizaron la estabilidad e inestabilidad atmosférica mediante la comparación del gradiente vertical de temperatura con la adiabática seca, y la inestabilidad potencial en proceso de levantamientos de capas, analizándose la distribución vertical de la temperatura potencial equivalente. La distribución altitudinal de las precipitaciones pronosticadas indicaron comportamiento de aumento hasta una altitud aproximada de $1500 \mathrm{~m}$ y de disminución por encima de esta altitud, los datos observados confirmaron el mismo comportamiento de variación altitudinal para el día 23 de marzo; los análisis del viento indicaron presencia de sistemas del Pacífico (flujos de barlovento) hasta aproximadamente $2500 \mathrm{~m}$ de altitud; los gradientes térmicos verticales mostraron atmósfera ligeramente estable en la zona de estudio; sin embargo la distribución vertical de la temperatura potencial equivalente mostró atmósfera potencialmente inestable sobre localidades asociados con aumento altitudinal de la precipitación. En consecuencia la presencia de sistemas del Pacífico o flujos de barlovento, la topografía y la inestabilidad potencial de la atmósfera constituyeron los forzantes de las precipitaciones ocurridas.
\end{abstract}

Palabras clave: Precipitación; pronóstico de precipitación; BRAMS; huaicos; Chosica.

\begin{abstract}
At present there are numerical models to provide information which favors the analysis and research in areas without meteorological observed data. The BRAMS regional model has forecast data from the global CPTEC model that should be used for the dynamic downscaling process whose results are useful in the analysis of predicted weather conditions in high-risk climatic event areas in order to prevent the negative effects and to use the predictive capacity of the model. This work was done using the BRAMS model to determine the origin of rainfall that caused the mudslides in Chosica in March 2015. For that purpose the altitudinal histograms distribution of forecasted precipitation data with five days in advance and observed data at meteorological stations was determined; also were analyzed surface winds and height winds, to identify associated flows (Pacific systems or the East systems) with precipitation; also the atmospheric stability and instability were analyzed by comparing the vertical temperature lapse rate with the dry adiabatic lapse rate, and potential instability in the process of surveying layers, by analyzing the vertical distribution of equivalent potential temperature. The altitudinal distribution of predicted rainfall (histograms) showed increase behavior until an altitude of $1500 \mathrm{~m}$ and decrease behavior above this altitude level, the histograms of the registered data confirmed the same behavior of altitudinal variation for March 23; the surface and height wind analysis indicated presence of Pacific systems (windward flows) to about $2500 \mathrm{~m}$ altitude; in the analysis of stability and instability by comparing the lapse rates the study area showed slightly stable atmosphere; however the vertical distribution of the equivalent potential temperature showed potentially unstable atmosphere over areas associated with altitude increase precipitation. Consequently the presence of Pacific systems or windward flows, topography and potential instability of the atmosphere were the forcing factors the rainfall occurred.
\end{abstract}

Keywords: Precipitation; precipitation forecast; BRAMS; mudslides; Chosica. 


\section{Introducción}

El temor de la ocurrencia de huaicos en Chosica suele presentarse verano tras verano, debido principalmente a la mayor presencia de fenómenos convectivos, a lo largo de la costa y sierra central del Perú. Las precipitaciones excepcionales aunadas con la morfología y relieve de Chosica, material inconsolidado y carencia de vegetación y con la irresponsabilidad o imprudencia de la población al construir sus viviendas en los cauces naturales de las quebradas, generan remociones que al discurrir por los cauces traen consigo pérdidas socioeconómicas (Villacorta et al., 2015). Debido a ello se ve la necesidad de realizar estudios encaminados a sus pronósticos. Para los cuales son necesarios contar con informaciones meteorológicas, en especial de precipitación, de calidad y de una buena densidad de estaciones (Dillon et al., 2013, García Skabar et al., 2012).

La disponibilidad y densidad de estaciones, en gran parte del Perú, que nos brinden datos meteorológicos que ayuden a realizar estudios de diferentes eventos, es muy escasa. Sin embargo en la actualidad existen modelos numéricos del tiempo que nos permiten disponer de dicha información para el análisis e investigaciones (Dillon et al., 2013, García Skabar et al., 2012). Estos modelos nos permiten obtener datos de la serie histórica y de pronósticos.

Existen datos de pronóstico procedentes de los modelos globales para ser utilizados con modelos regionales como BRAMS, WRF, entre otros, sin embargo aún no se están haciendo uso en gran parte de Sud América y menos en Perú; esta información debería ser usada en el monitoreo de las condiciones de tiempo pronosticado para zonas con eventos climáticos de alto riesgo, con el fin de prevenir sus efectos negativos y así como también aprovechar la utilidad del modelo de predecir estos eventos (Dillon et al., 2013). Esta práctica permitiría ir evaluando el desempeño del modelo. Debido a ello en este estudio se han usado precipitaciones pronosticados por el modelo BRAMS (2007) para el análisis de su comportamiento, y los forzantes que los originaron durante el evento del huaico ocurrido en Chosica el 23 de Marzo del 2015.

Por todo lo antes mencionado, en este trabajo se planteó los siguientes objetivos: Determinar la distribución altitudinal de la precipitación pronosticada, en la cuenca del Rimac. determinar la distribución altitudinal de la precipitación registrados en la cuenca del Rimac y determinar los factores forzantes de las precipitaciones asociadas al huaico ocurrido.

\section{Materiales y métodos Datos y software}

En esta investigación se usaron datos de pronósticos cada 6 horas para un horizonte de 8 días del modelo global de CPTEC (http://brams.cptec.inpe.br/ ), para el período del 18 al 26 de marzo de 2015, debido a que el huayco en Chosica ocurrió el día 23 de marzo; la información tiene una resolución temporal de 6 horas y una resolución espacial de $110 \mathrm{~km}$. O sea, para cada día existen información a las 00Z, 06Z, 12Z y 18Z donde para el primer día la información de $00 Z$ son datos de condiciones iniciales. Las variables meteorológicas del modelo global necesarias para el BRAMS son altura geopotencial (hgt), temperatura $(\mathrm{T})$, humedad relativa (HR), viento zonal (u) y viento meridional (v).

Por otro lado, también se usaron datos registrados de precipitación de las estaciones meteorológicas del Servicio Nacional de Meteorología e Hidrología (SENAMHI, 2015) Chosica, Matucana, San Mateo de Huanchor, Rio Blanco y Casapalca y, de la Universidad Nacional Agraria La Molina "UNALM" (Von Humboldt).

Los softwares usados en el estudio fueron: modelo BRAMS 4.2, Office, programa GrADS y Google Earth.

\section{Área de estudio}

La zona donde se realizó la investigación comprende la cuenca del Río Rimac, desde el Océano Pacífico hasta Casapalca (Figura 2).

\section{Metodología}

Determinación de la distribución altitudinal de la precipitación con datos de pronóstico.

Corrida del modelo. Los datos pronosticados del modelo global primeramente fueron convertidos para poder ser utilizados en el modelo regional BRAMS; luego se vio la conFiguración del modelo y en este caso se utilizó la conFiguración temporal de una hora y conFiguración horizontal de tres dominios, como se observa en la Figura 1, el dominio G1 o dominio padre de $64 \mathrm{~km}$, los dominios anidados G2 de $16 \mathrm{~km}$ y el tercer dominio G3 de $2 \mathrm{~km}$.; en seguida se realizó el pre procesamiento para la generación de condiciones de superficie y condiciones de frontera; luego se continuó con la corrida o procesamiento y culminándose con el post procesamiento de sus salidas. El estudio se hizo con las salidas del tercer dominio.

Elección de los puntos de análisis. Para el análisis de las salidas del BRAMS primero se eligieron 10 puntos a lo largo de la cuenca, considerando que la distribución comprenda desde el océano hasta Casapalca, con la finalidad de abarcar todo el área de estudio, los cuales se muestran en la Tabla 1 y Figura 2. Para la obtención de los datos en los puntos elegidos, el software utilizado suministra la información de la coordenada más próxima.

Análisis de la distribución altitudinal. Los datos de precipitación obtenidos en el punto anterior fueron exportados, haciendo uso del GRADS, para una hoja de cálculo. Luego se realizaron el análisis temporal cada 3 horas de los histogramas respectivos para los días 22, 23, 24 y 25. Estos histogramas permitieron identificar los flujos que determinaron la precipitación, si fueron del Este o del Oeste, si los histogramas indican aumento hasta una cierta altitud y luego una disminución se dirá que fueron sistemas del Pacífico, caso contrario se considerará como sistemas del Este. Además la existencia de estos 
Tabla 1. Ubicación de los 10 puntos referenciales seleccionados para el análisis de distribución altitudinal de precipitación

\begin{tabular}{lcccccccccc}
\hline & Océano & $\begin{array}{c}\text { Santa } \\
\text { Anita }\end{array}$ & Naña & Chacla-cayo & Chosica & San Bar-tolomé & Matu-cana & $\begin{array}{c}\text { San } \\
\text { Mateo }\end{array}$ & Chicla & Casa-palca \\
\hline Latitud $\left({ }^{\circ} \mathrm{S}\right)$ & 12,08 & 12,03 & 11,99 & 11,95 & 11,92 & 11,91 & 11,84 & 11,75 & 11,71 & 11,64 \\
Longitud $\left({ }^{\circ} \mathrm{W}\right)$ & 77,12 & 76,97 & 76,84 & 76,74 & 76,69 & 76,52 & 76,37 & 76,3 & 76,26 & 76,24 \\
Altitud (m) & 1 & 260 & 525 & 888 & 1167 & 1468 & 2576 & 3181 & 3829 & 4258 \\
\hline
\end{tabular}

\section{$64 \mathrm{~km}$}

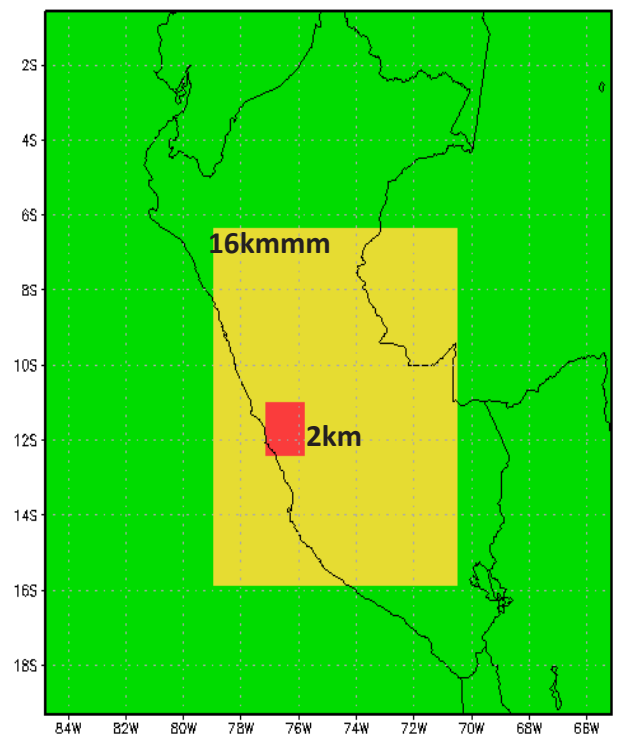

Figura 1. ConFiguración del modelo utilizado en el estudio. Dominio $1(64 \mathrm{~km})$, dominio 2 (16km) y dominio 3 (2 $\mathrm{km})$

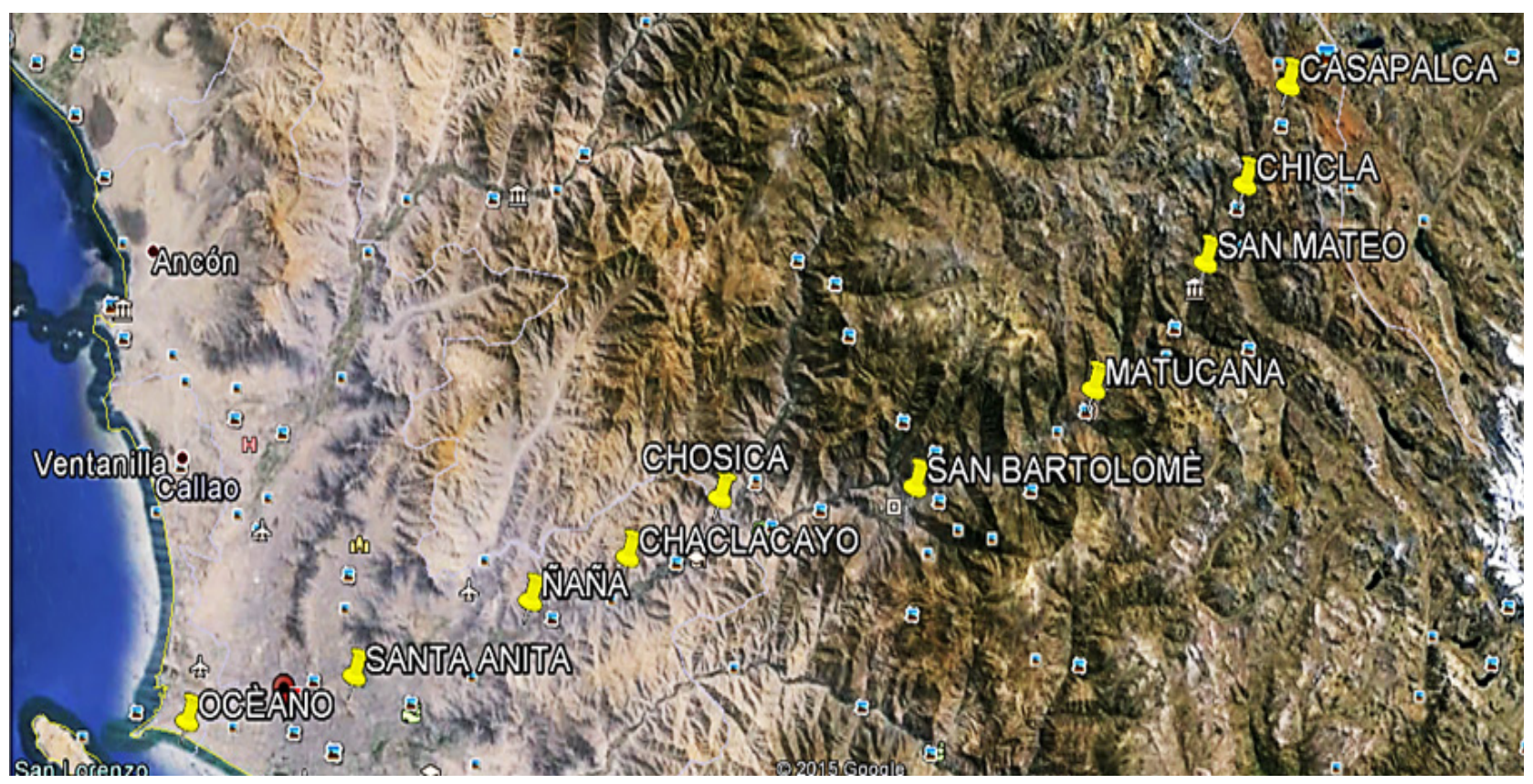

Figura 2. Ubicación de los 10 puntos seleccionados 
histogramas serán los indicadores que las precipitaciones fueron pronosticadas con 5 días de anticipación para el caso del día 23

\section{Determinación de la distribución altitudinal de la precipitación con datos registrados.}

Con datos de precipitación diaria de seis estaciones meteorológicas seleccionadas (Tabla 2) se realizaron los histogramas respectivos del 22 al 25 de marzo con la finalidad de ver si guardan el mismo comportamiento que los datos pronosticados por el modelo BRAMS.

\section{Determinación de los factores forzantes de las precipitaciones asociadas al evento}

Para determinar estos forzantes se han analizado los flujos en superficie y en altura, así como la estabilidad e inestabilidad de la atmósfera en tres lugares Océano, Chosica y Matucana cuyas ubicaciones se muestran en la Tabla 1 y Figura 2, elegidos teniendo en cuenta como punto de referencia a Chosica, una a menor altitud y otra a mayor altitud.

Con el viento resultante del tercer dominio se analizó los flujos en niveles de $24 \mathrm{~m}, 731 \mathrm{~m}, 2680 \mathrm{~m}$ y $3266 \mathrm{~m}$ para identificar si son de barlovento o sotavento; estos flujos fueron superpuestos al campo de precipitación y topografía. El análisis se realizó cada seis horas para el día 23 y para los días 22,24 y 25 , solo para las $18 \mathrm{Z}, 18 \mathrm{Z}$ y $15 \mathrm{Z}$, respectivamente. Si en estos análisis han de observarse flujos del Pacífico, o sea flujos de barlovento, en los niveles bajos, serán los indicadores que fueron los responsables de las precipitaciones.

En lo que concierne a la inestabilidad y estabilidad atmosférica, en los puntos elegidos se procedió a determinar los perfiles térmicos para los días 22, 23, 24 y 25, cada seis horas, con la finalidad de obtener los gradientes térmicos verticales $(\gamma)$. Una vez obtenidos, se compararon con el gradiente adiabática seco $\left(\Gamma=0.01^{\circ} \mathrm{C} / \mathrm{m}\right)$ y poder decir si la atmósfera fue estable $(\gamma<\Gamma)$ o inestable $(\gamma>\Gamma)$. La atmósfera será inestable cuándo el gradiente térmico vertical es más intenso que la adiabática seca.

Para el análisis del levantamiento de capas se determinó la estabilidad e inestabilidad potencial mediante el perfil vertical de la temperatura potencial equivalente $(\theta \mathrm{e})$, para lo cual se realizó un corte transversal a lo largo de la latitud de $11.92^{\circ} \mathrm{S}$ desde $77.12^{\circ} \mathrm{W}$ hasta $66.2^{\circ} \mathrm{W}$, y una extensión vertical hasta 3100 metros, se eligieron estas altitudes para apreciar la variable en los tres puntos a la vez (Océano, Chosica y Matucana). Se dirá que la atmósfera es potencialmente inestable o que presenta inestabilidad mecánica si se aprecia disminución de la $\theta$ e con la altitud, caso contrario se considerará estable, también se considerará potencialmente neutral en el caso de que la $\theta \mathrm{e}$ no presente variación con la altura.

Para la determinación de la temperatura potencial equivalente $(\theta \mathrm{e})$ se tuvo en consideración la siguiente fórmula:

$\theta e=\theta \exp \left(\frac{L w s}{c p T}\right)$

Donde:

$\theta$ : Temperatura potencial $(\mathrm{K})$

L: Calor latente de condensación (cal/gr)

ws: Relación de mezcla de saturación (gr/gr)

cp: Calor específico a presión constante $\left(\mathrm{cal} / \mathrm{gr} .{ }^{\circ} \mathrm{C}\right)$

$\mathrm{T}$ : Temperatura del aire cuando se alcanza la saturación por primera vez $\left({ }^{\circ} \mathrm{C}\right)$

\section{Resultados y discusión}

Determinación de la distribución altitudinal de la precipitación con información pronosticada.

Según la Figura 3, los histogramas muestran las mismas características a lo largo del día y en los 4 días; de océano a se observa variación altitudinal de aumento y de San Bartolomé (1468m) a Chicla (3829m) y Casapalca (4258 m) la precipitación disminuye con la altitud; la otra característica resaltante es que en los puntos extremos océano $(0 \mathrm{~m})$ y Casapalca $(4258 \mathrm{~m})$, el modelo pronosticó $0 \mathrm{~kg} / \mathrm{m}^{2}$ para los días 22, 23, 24 y 25; en la altitud de San Bartolomé se presentó los valores más altos, $160 \mathrm{~kg} /$ $\mathrm{m}^{2}$ el día 22 y $330 \mathrm{~kg} / \mathrm{m}^{2}$ el día 25 ; en Chosica los valores variaron de $80 \mathrm{~kg} / \mathrm{m}^{2}$ para el día 22 y $140 \mathrm{~kg} / \mathrm{m}^{2}$ el día 25 . La primera característica indica que durante el evento las precipitaciones se dieron en altitudes bajas por debajo de los $1468 \mathrm{~m}$, o sea en lugares con características de suelos inconsistentes propicios para la formación de huaycos; por otro lado teniendo en cuenta que el modelo no está calibrado ni validado las magnitudes de las precipitaciones solo deben considerarse como referenciales, pero útiles como indicadores de la ocurrencia de la precipitación. Finalmente su característica de aumento desde el Océano hasta San Bartolomé, y de disminución después de este punto hasta Casapalca nos permite inferir que esa precipitaciones fueron originados por los flujos ascendentes desde el Pacífico esto es, flujos de barlovento. Considerando que el modelo se inicializó con datos de $00 \mathrm{Z}$ del día 18 de marzo,

Tabla 2. Ubicación de las 6 estaciones meteorológicas tomadas en cuenta para el análisis altitudinal de precipitación

\begin{tabular}{lcccccc}
\hline & Von Humboldt & Chosica & Matucana & San Mateo & Rio Blanco & Casapalca \\
Latitud ( 'S) & 12,08 & 11,92 & 11,83 & 11,75 S & 11,73 & 11,63 \\
\hline Longitud ( 'W) & 76,93 & 76,68 & 76,37 & 76,3 & 76,25 & 76,18 \\
Altitud (m) & 246 & 906 & 2431 & 3182 & 3550 & 4100 \\
\hline
\end{tabular}




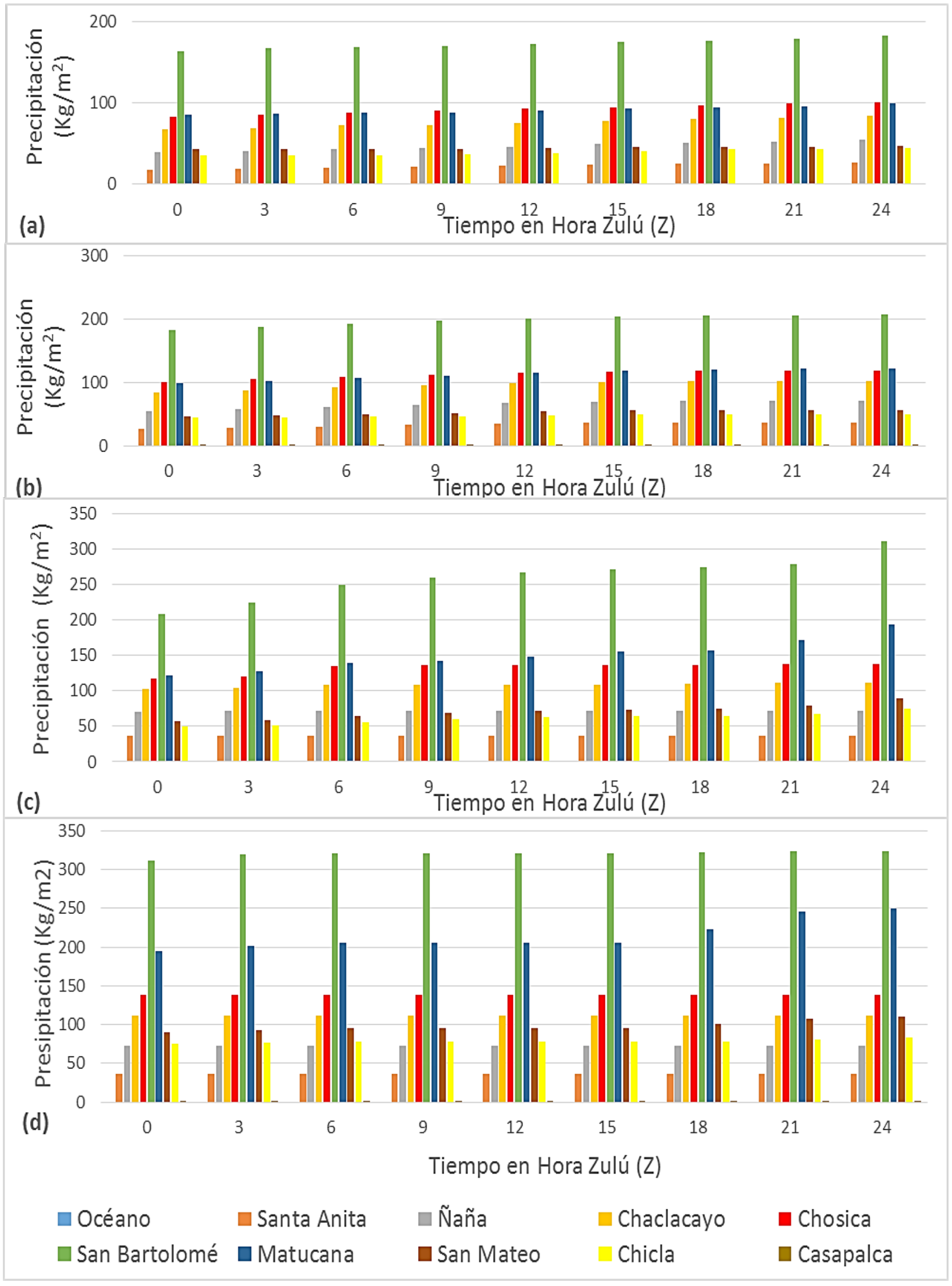

Figura 3. Histogramas de precipitaciones pronosticadas para los días 22 (a), 23 (b), 24 (c) y día 25 (d) de marzo, respectivamente 
las precipitaciones estuvieron pronosticados con cinco días de anticipación para el día 23 de marzo de 2015.

\section{Determinación de la distribución altitudinal de la precipitación con datos registrados.}

Según la Figura 4, los histogramas no muestran una tendencia común entre los cuatro días de análisis, la característica común es la ausencia de precipitación en Océano; el día 22 se registraron precipitaciones por debajo de $5 \mathrm{~mm}$, este va en aumento desde Océano hasta Matucana y luego disminuye hasta Rio blanco y finalmente presenta un valor máximo en Casapalca; el día 23 se registró el máximo valor de precipitación en Chosica de cerca de los $20 \mathrm{~mm}$, este también fue el máximo valor de todo el período de estudio, a partir del cual disminuyen hacia Casapalca; para el día 24 no hubo registró en Chosica, los mayores valores se encontraron entre Matucana, San Mateo y Río Blanco, de alrededor de $10 \mathrm{~mm}$, siendo mayor en este último; para el día 25 tampoco se registraron datos en Chosica, y la tendencia va en aumento desde Matucana hasta Casapalca, alcanzando un valor máximo de alrededor de $8 \mathrm{~mm}$. Según estos histogramas, salvo para el día 24 y 23 , no se observa relación directa con los histogramas pronosticados.

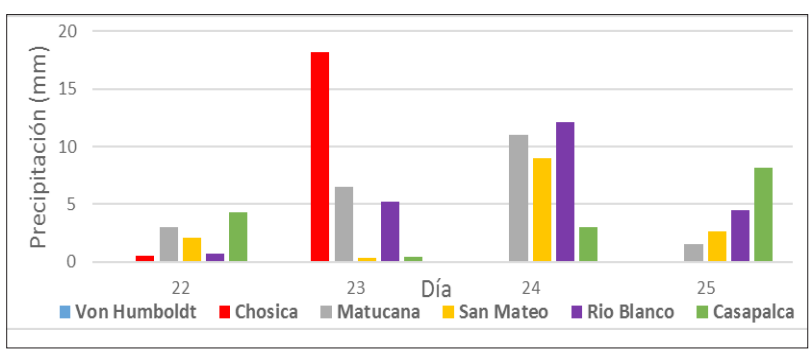

Figura 4. Histogramas de precipitación según datos observados

\section{Determinación de los factores forzantes de las precipitaciones asociadas al evento}

Las Figuras 5 y 6 muestran a detalle el flujo del viento en niveles bajos (a) y en niveles altos (b) para las horas analizadas el día 23. En el Anexo (d) se presentan Figuras iguales correspondientes a las horas de análisis del 22 al 25.

Las Figuras 7 y 8 muestran los resultados para los análisis de los forzantes concernientes a los flujos próximos a la superficie y en altura. Las áreas oscuras de color rojo constituyen la distribución espacial de la precipitación pronosticada por el modelo (para las horas indicadas) y se observa que se concentran en la partes bajas a lo largo de la cuenca. Por otro lado las flechas que se observan constituyen los flujos de viento en los diferentes niveles. Las Figuras también muestran las isolíneas de la topografía. La Figura 7 indica la presencia de precipitación considerable en la zona de análisis, aunque no exactamente en Chosica pero sí cercana a esta; se aprecia también flujos de vientos con predominancia de sistemas del pacífico en los dos primeros niveles ( $24 \mathrm{~m}$ y $731 \mathrm{~m}$ ) a las $3 \mathrm{Z}, 9 \mathrm{Z}$ y $15 Z$, y predominancia de los sistemas del Este en los dos niveles siguientes $(2680 \mathrm{~m}$ y $3266 \mathrm{~m})$; y a las $21 \mathrm{Z}$ se observa intensificación de los flujos del oeste en los dos primeros niveles y desaparición de los Estes en los niveles siguientes.

La Figura 8 muestra flujos a las 18Z, 21Z, $18 \mathrm{Z}$ y $15 \mathrm{Z}$ para los días 22, 23, 24 y 25, respectivamente, se observa un incremento de la cantidad de precipitación desde el 22 hasta el 25, el cual se identifica por la intensificación de los colores rojo-oscuros. Los flujos variaron en intensidad en los diferentes días, así como también sus conFiguraciones fueron distintas en los diferentes niveles y días; para el día 22 en los dos primeros niveles fueron del oeste y no fueron muy intensos, en los dos últimos niveles fueron del este y más intensos; para el día 23 en los cuatro niveles fueron del oeste, más intensos en los dos primeros niveles; para el día 24 fueron marcadamente predominantes del Oeste en los cuatro niveles, los flujos de los dos primeros niveles fueron menos intensos que el caso anterior; en el día 25 hubo gran intensificación tanto de los Estes y de los Oestes en los dos primeros niveles, y en los dos niveles superiores se aprecia encuentro de los dos sistemas, debido a que no se puede diferenciar la influencia de algún tipo de flujo. En consecuencia de acuerdo a las Figuras 5 y 6 se afirma que uno de los forzantes de esas precipitaciones fueron los sistemas del pacífico.

Finalmente la Figura 9 muestran los diferentes perfiles térmicos en los tres lugares considerados, para las 06, 12 , 18 y 24 z del día 22 al 25; los gradientes oscilan entre 0.005 ${ }^{\circ} \mathrm{C} / \mathrm{m}$ y $0.009{ }^{\circ} \mathrm{C} / \mathrm{m}$, la intensidad de estos gradientes no superan a la intensidad del gradiente adiabático seco (0.01 ${ }^{\circ} \mathrm{C} / \mathrm{m}$ ) los que indican que el evento estuvo asociado con una atmósfera ligeramente estable. En océano se obtuvieron los valores más intensos iguales a $0.009^{\circ} \mathrm{C} / \mathrm{m}$ en los días 22, 24 y 25; en Chosica se encontraron los valores menos intensos desde $0.006{ }^{\circ} \mathrm{C} / \mathrm{m}$ en los días 22 y 24 a $0.005^{\circ} \mathrm{C} / \mathrm{m}$ el día 23, y en Matucana se encontró el valor de $0.008^{\circ} \mathrm{C} / \mathrm{m}$ para todos los días. De acuerdo a estos resultados se indica que la estabilidad e inestabilidad actuó como un forzante negativo para las precipitaciones ocurridas.

Las Figuras 10, 11, 12 y 13 muestran resultados del análisis de estabilidad e inestabilidad en el proceso de levantamiento de capas debido a convección orográfica de los flujos del Pacífico- La temperatura potencial equivalente está representada por las isolíneas de colores, los que tienden al amarillo y al rojo representan valores altos y los que tienden al azul y al morado representan valores bajos. Se tiene que tener en cuenta que el Océano esta alrededor de los $77.1^{\circ} \mathrm{W}$, Chosica alrededor de los $76.7^{\circ} \mathrm{W}$ y Matucana alrededor de los $76.3^{\circ} \mathrm{W}$, así como también que si la temperatura potencial equivalente disminuye con la altura indica inestabilidad o atmósfera potencialmente inestable y caso contrario se considerará potencialmente estable.

En la Figura 10, muestra disminución con la altura de la temperatura potencial equivalente para las 4 


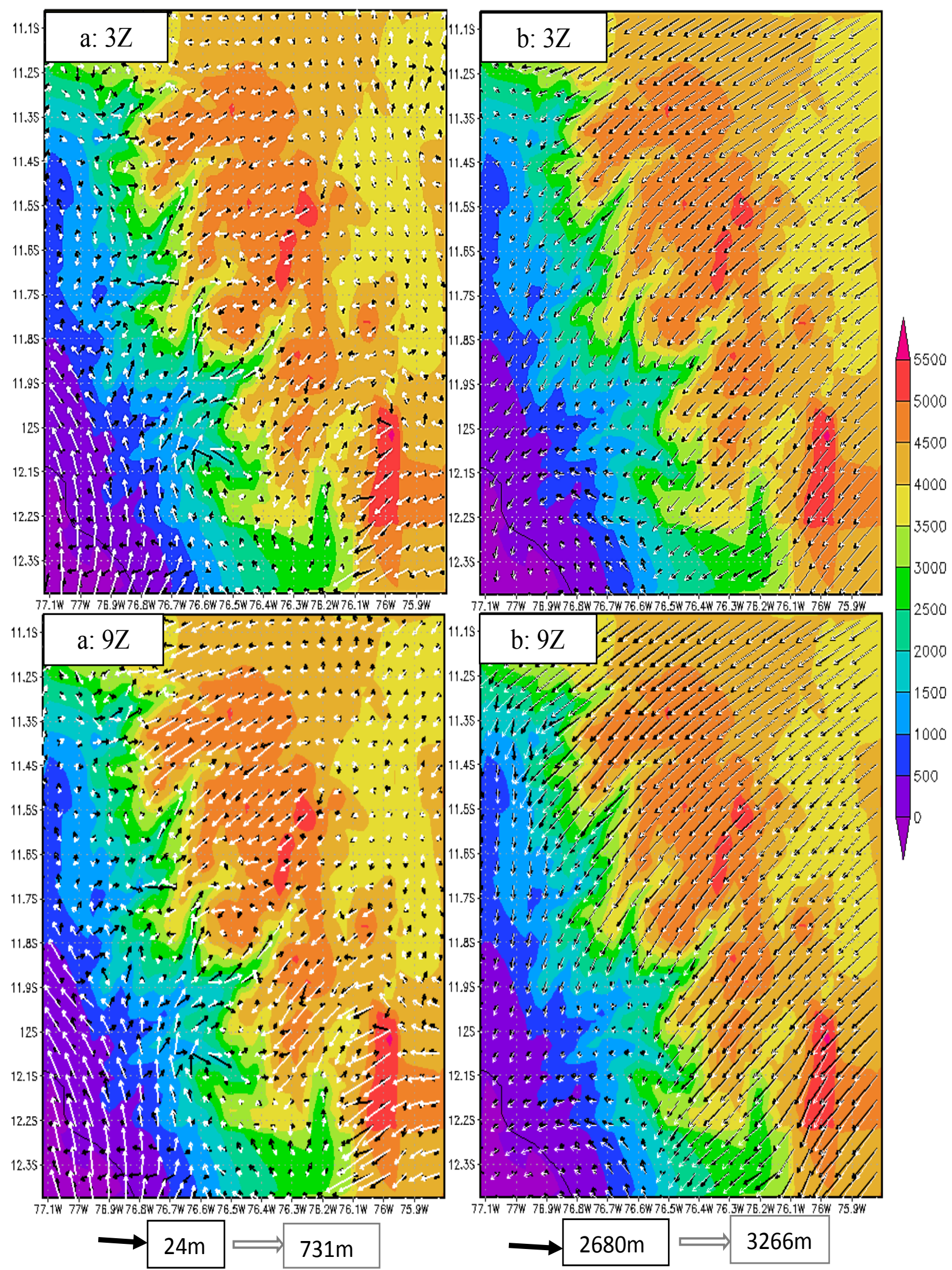

Figura 5. Campo de viento en cuatro niveles: $24 \mathrm{~m}$ y $731 \mathrm{~m}$ (a), 2680m y $3266 \mathrm{~m}$ (b) superpuesto sobre la topografía (áreas sombreadas) para el día 23 a las horas 3 y $9 \mathrm{Z}$ 


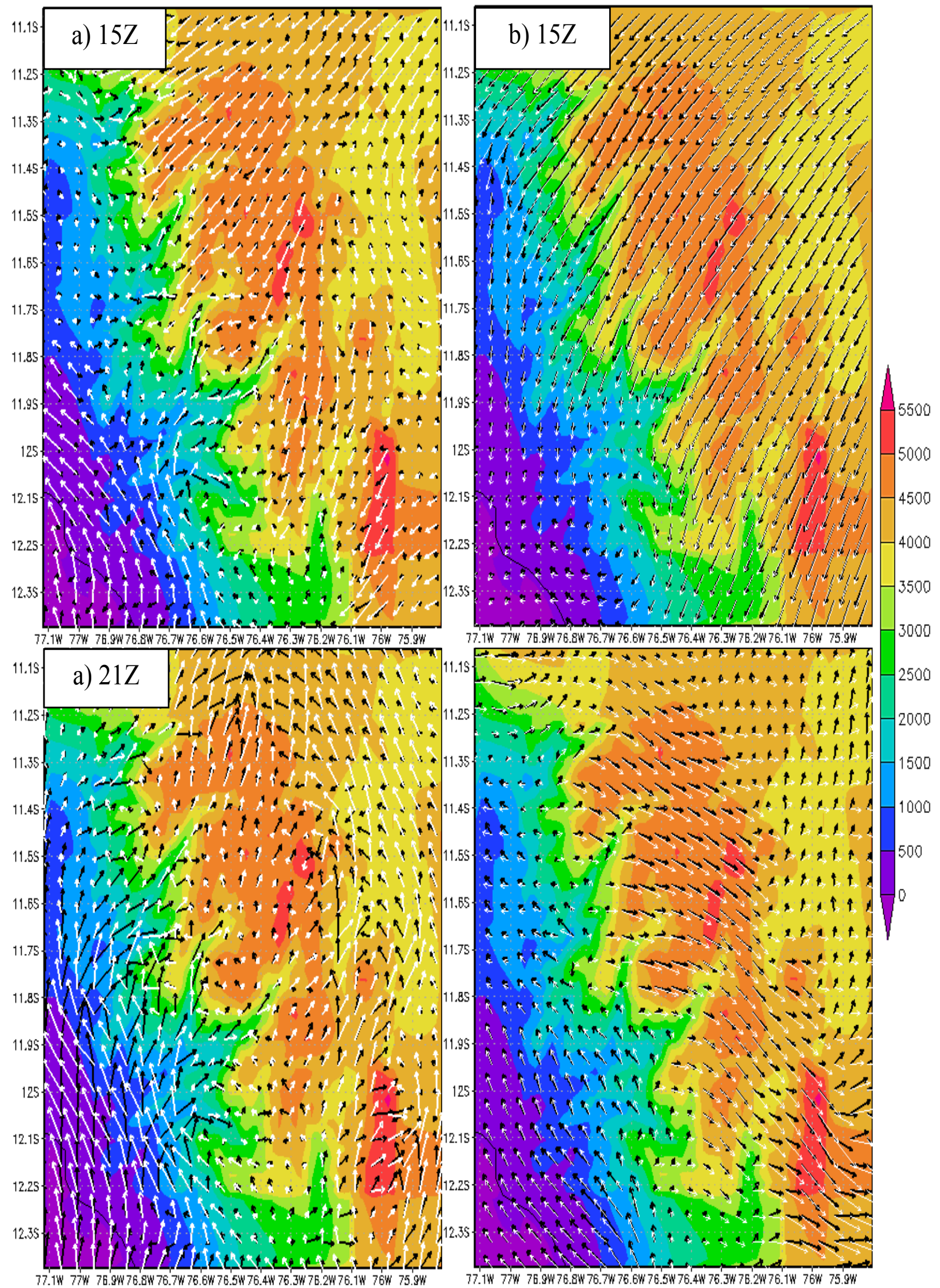

Figura 6. Campo de viento en cuatro niveles: $24 \mathrm{~m}$ y $731 \mathrm{~m}$ (a), $2680 \mathrm{~m}$ y $3266 \mathrm{~m}$ (b) superpuesto sobre la topografía (áreas sombreadas) para el día 23 a las horas 15 y $21 Z$ 

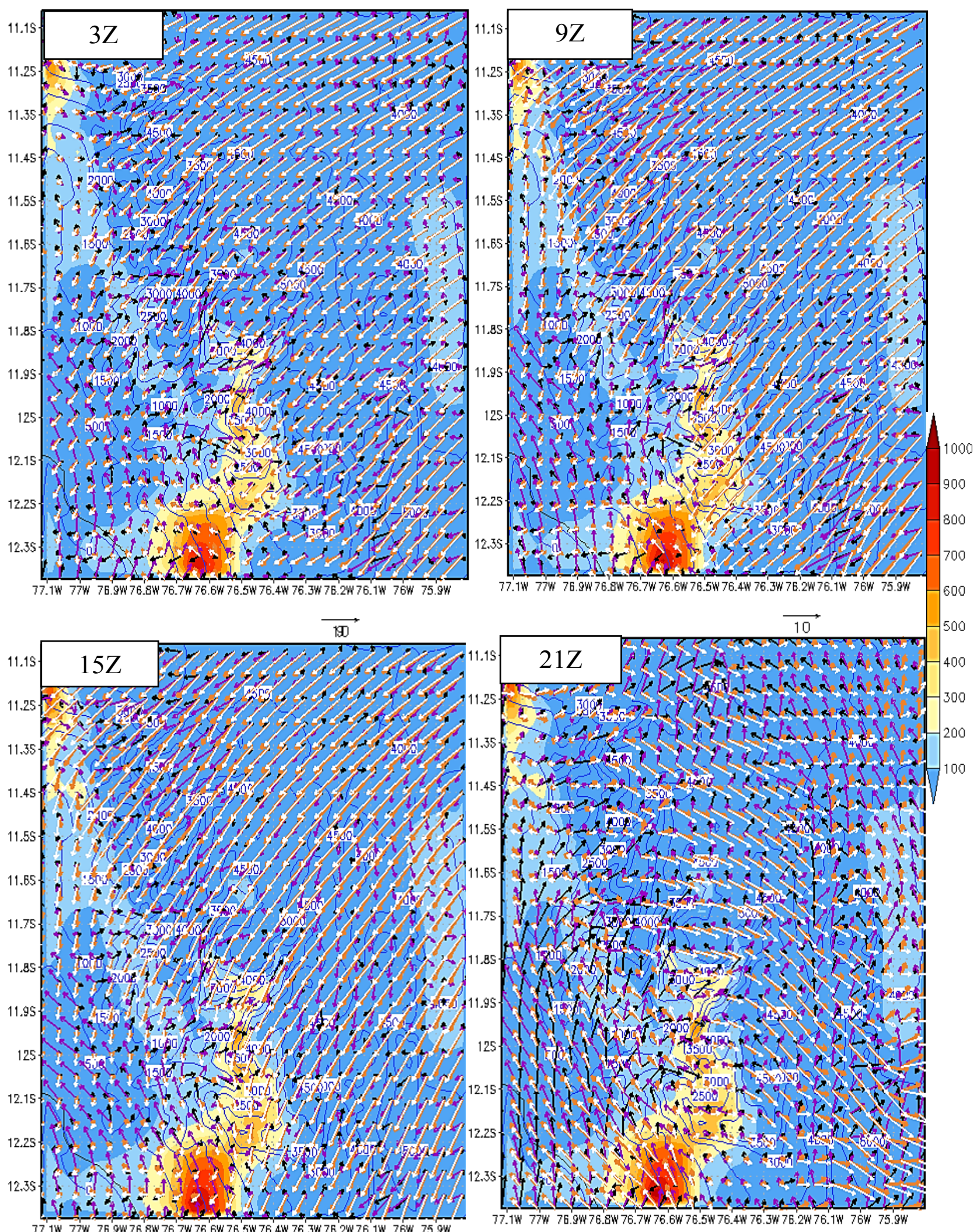

212 f

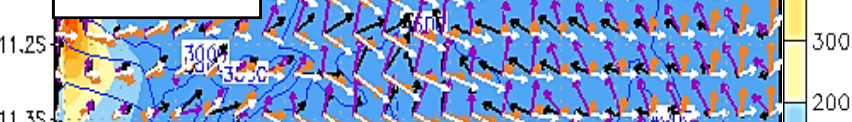

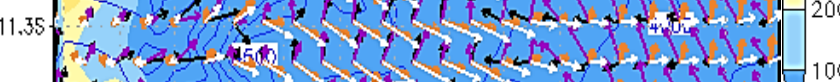

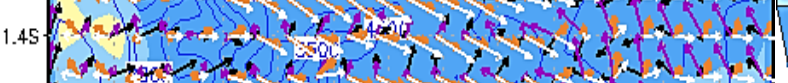

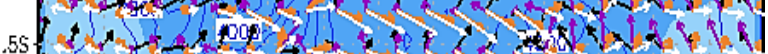

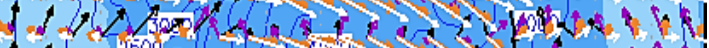

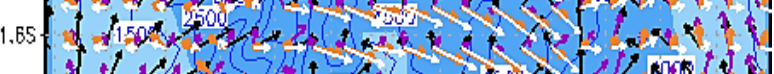

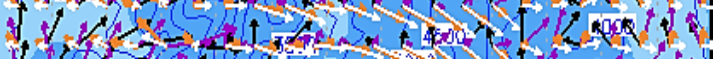

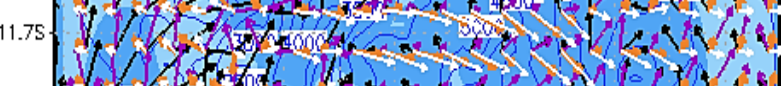

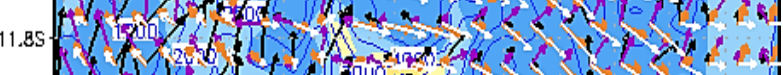

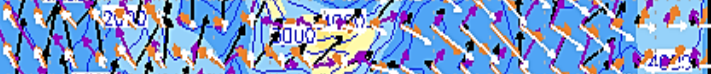

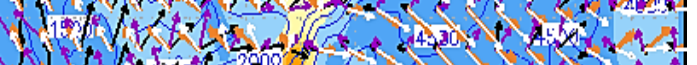

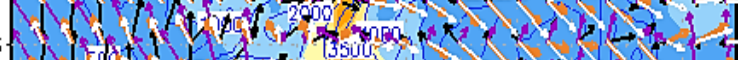

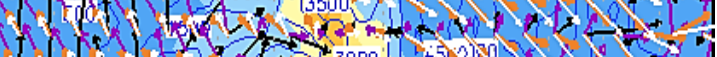
12.15 f

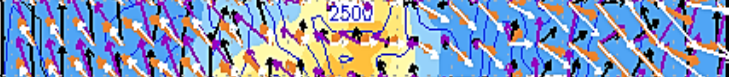

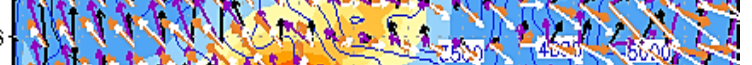

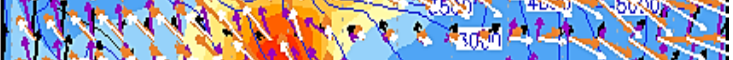

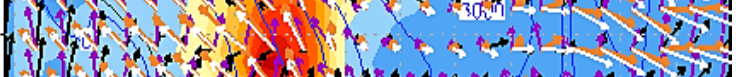
$77.1 \mathrm{~W} 77 \mathrm{~W} 76.9 \mathrm{~W} 76.8 \mathrm{~W} 76.7 \mathrm{~W} 76.6 \mathrm{~W} 76.5 \mathrm{~W} 76.4 \mathrm{~W} 76.3 \mathrm{~W} 7 \mathrm{6} .2 \mathrm{~W} 76.1 \mathrm{~W}$ 76W 75.9W

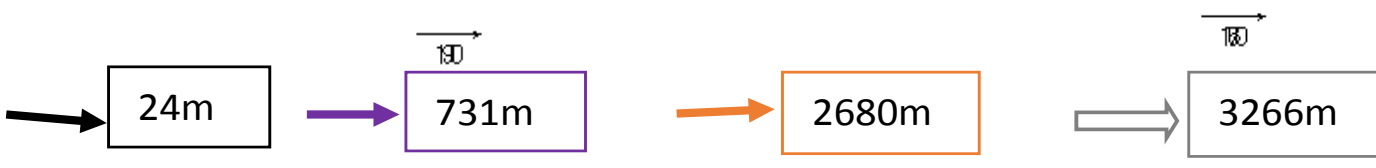

Figura 7. Campo de viento en cuatro niveles superpuesto a la precipitación en superficie para el día 23 a las horas $3 Z$, $9 \mathrm{Z}, 15 \mathrm{Z}$ y $21 \mathrm{Z}$, respectivamente 

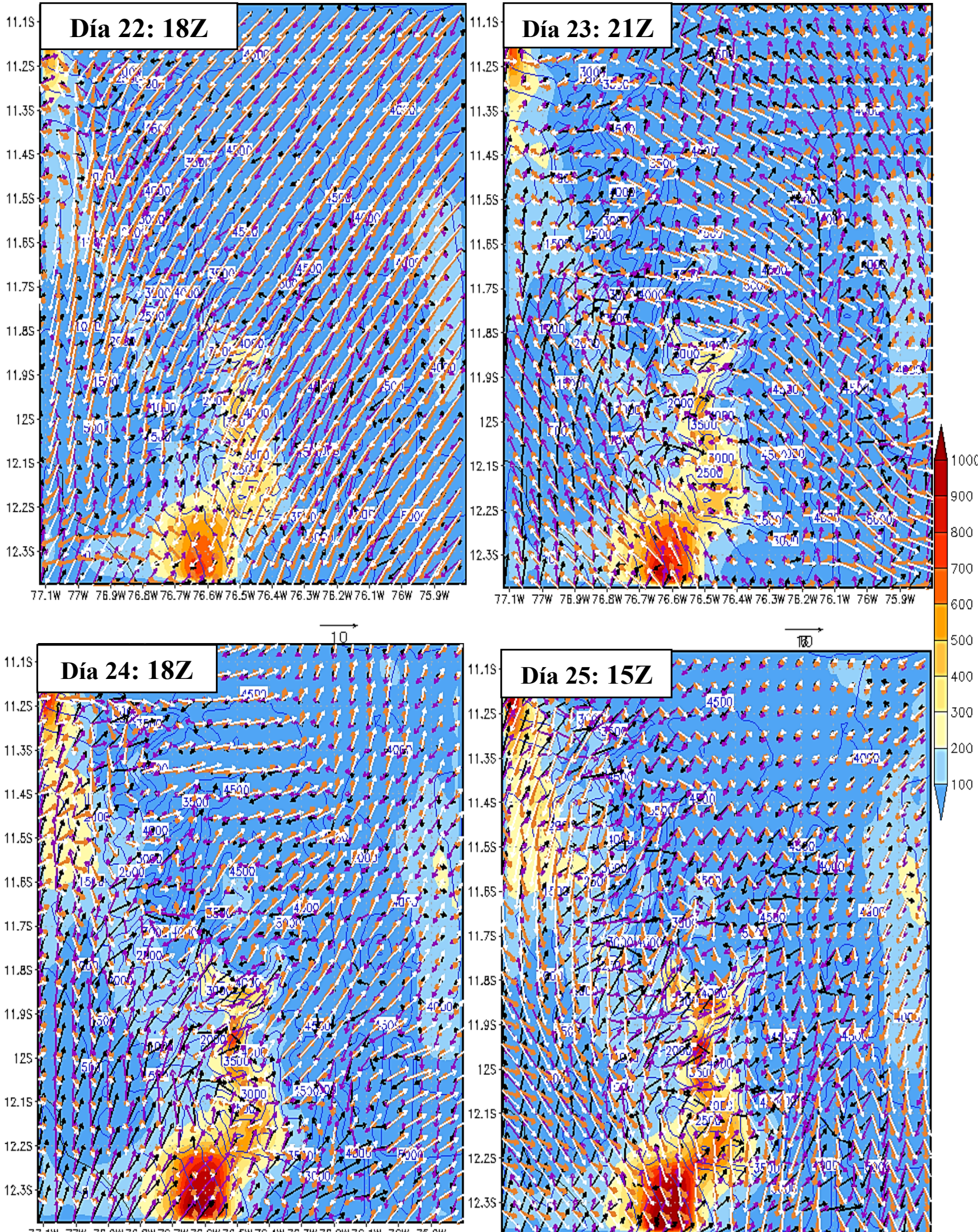

1.

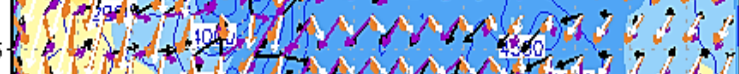

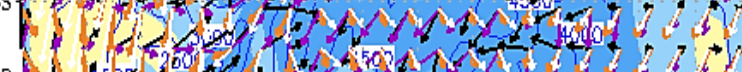

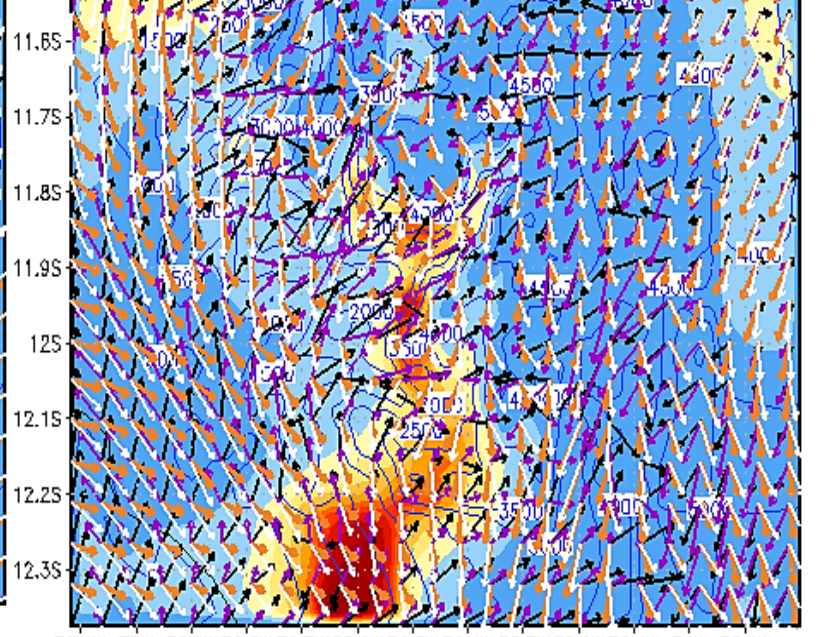

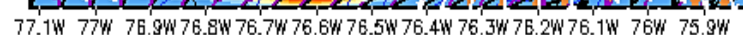

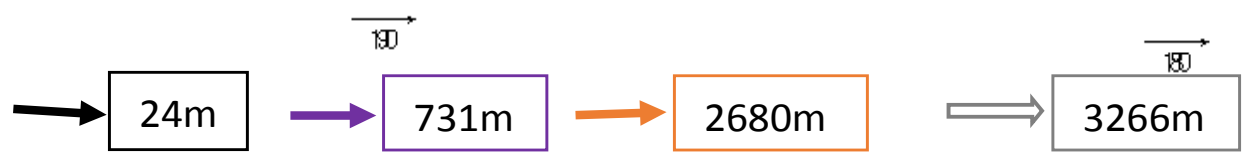

Figura 8. Campo de precipitación y flujos de vientos en niveles de $24,731,2680$ y $3266 \mathrm{~m}$ a las $18 \mathrm{Z}, 21 \mathrm{Z}, 18 \mathrm{Z}$ y $15 \mathrm{Z}$ para el día 22, 23, 24 y 25 respectivamente 


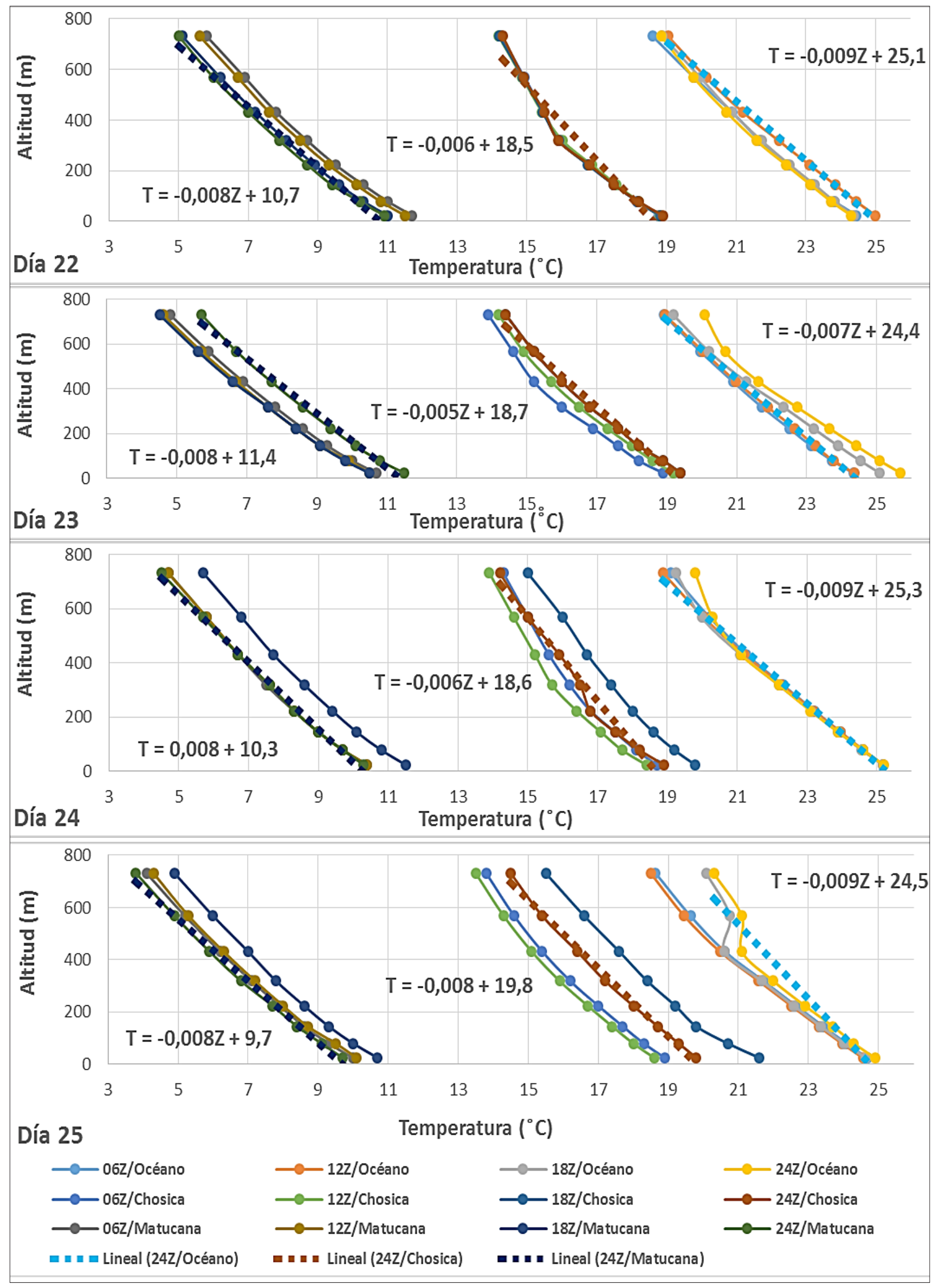

Figura 9: Perfiles de temperatura 
horas analizadas desde $77^{\circ} \mathrm{W}$ hacia el oeste y hasta aproximadamente $500 \mathrm{~m}$ de altura, tal distribución vertical indica atmósfera potencialmente inestable; desde $77^{\circ} \mathrm{W}$ a $76.4^{\circ} \mathrm{W}$ las isolíneas son cuasiverticales en la capa de $500 \mathrm{~m}$ los que indican atmósfera ligeramente inestable a potencialmente neutral; pero en el extremo este sobre Matucana las isolíneas indican aumento de temperatura potencial equivalente indicando atmósfera potencialmente estable.

La Figura 11 muestra características similares a la Figura 10 , en este caso la disminución con la altura ocurre desde $76.55^{\circ} \mathrm{W}$ hacia el oeste con extensión vertical de hasta $2200 \mathrm{~m}$ indicando así atmósfera potencialmente inestable para mayor área, la cual implica también aumento de inestabilidad sobre zona de Chosica a comparación del día 22; la zona de neutralidad y estabilidad se restringe a la parte este desde $76.55^{\circ} \mathrm{W}$ a $76.2^{\circ} \mathrm{W}$.

La Figura 12, para las $06 Z$ y $12 Z$ muestra que la inestabilidad potencial se restringió de $76.95^{\circ} \mathrm{W}$ hacia el oeste con una extensión vertical de unos $400 \mathrm{~m}$; el resto de la zona este muestra condiciones de estabilidad potencial. Mientras que para las $18 \mathrm{Z}$ y $24 \mathrm{Z}$ se aprecia la inestabilidad potencial se extendió hasta $76.55^{\circ} \mathrm{W}$ con una extensión vertical de aproximadamente $600 \mathrm{~m}$; el resto de la zona este se caracteriza por condiciones potencialmente neutral y estable afectando la zona de Matucana.

Según la Figura 13, a las $6 \mathrm{Z}$ y $12 \mathrm{Z}$ la disminución vertical de la temperatura potencial se restringe a la parte oeste desde los $76.95^{\circ} \mathrm{W}$ con extención vertical de $400 \mathrm{~m}$, el resto de la zona este presenta atmósfera potencialmente estable. Para las $18 \mathrm{Z}$ y $24 \mathrm{Z}$ las situaciones se modificaron, las condiciones inestabildad potencial se extendió hasta $76.5^{\circ} \mathrm{W}$ con su extensión vertical que alcanza aproximadamente los $700 \mathrm{~m}$. El resto de la zona este presenta atmósfera potencialmente estable.

\section{Conclusiones}

La distribución altitudinal de las precipitaciones pronosticadas indicaron comportamiento de aumento en los puntos ubicados a menor altitud de $1500 \mathrm{~m}$ y disminución por encima de este nivel altitudinal. Esta distribución de histogramas de precipitación son consecuencia de los forzantes de sistemas del Oeste, los cuales fueron pronosticados con aproximadamente una semana de

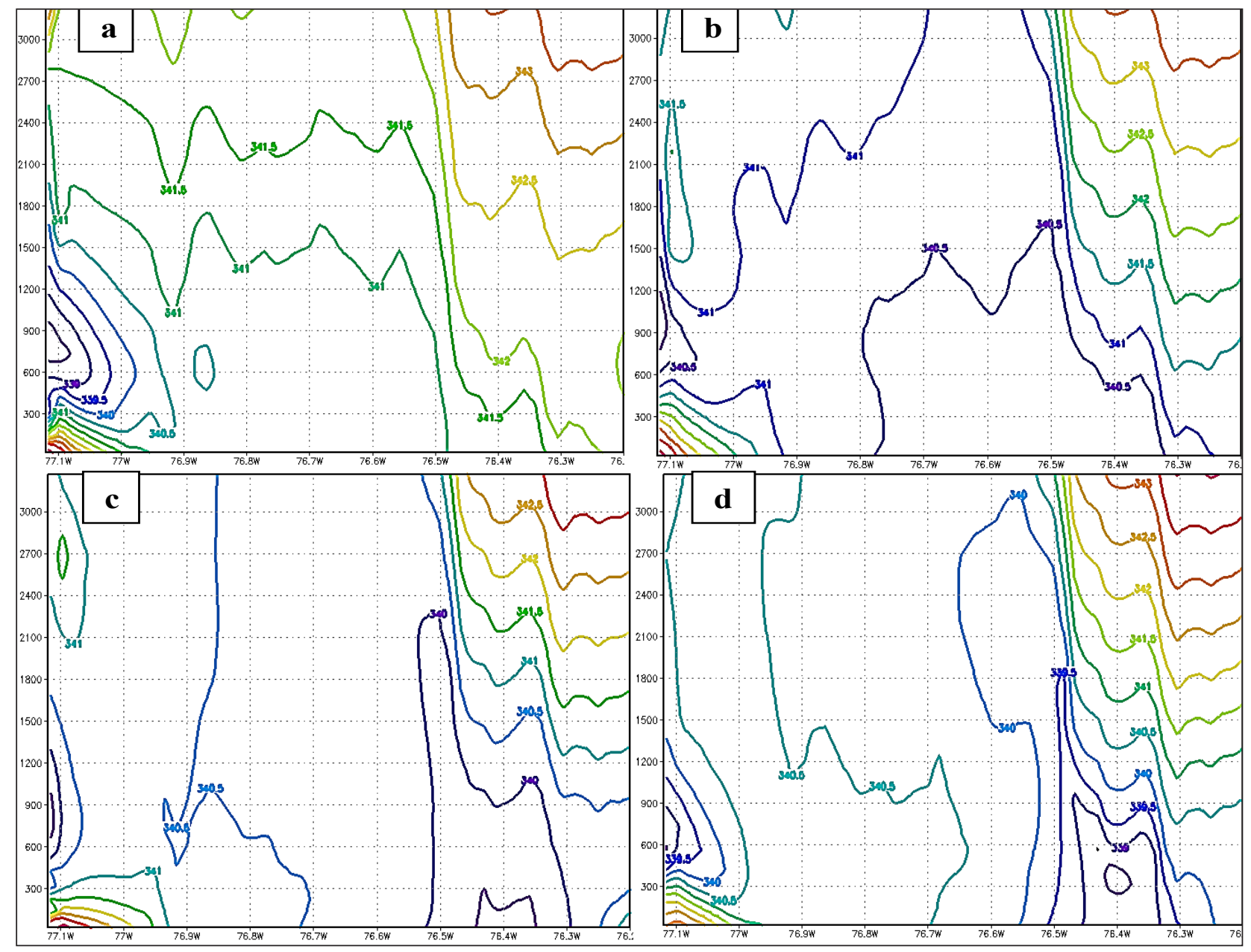

Figura 10. Distribución vertical de temperatura potencial equivalente para el día 22 de marzo para las horas $06 Z$ (a), $12 \mathrm{Z}$ (b), $18 \mathrm{Z}$ (c) y $24 \mathrm{Z}$ (d), respectivamente 


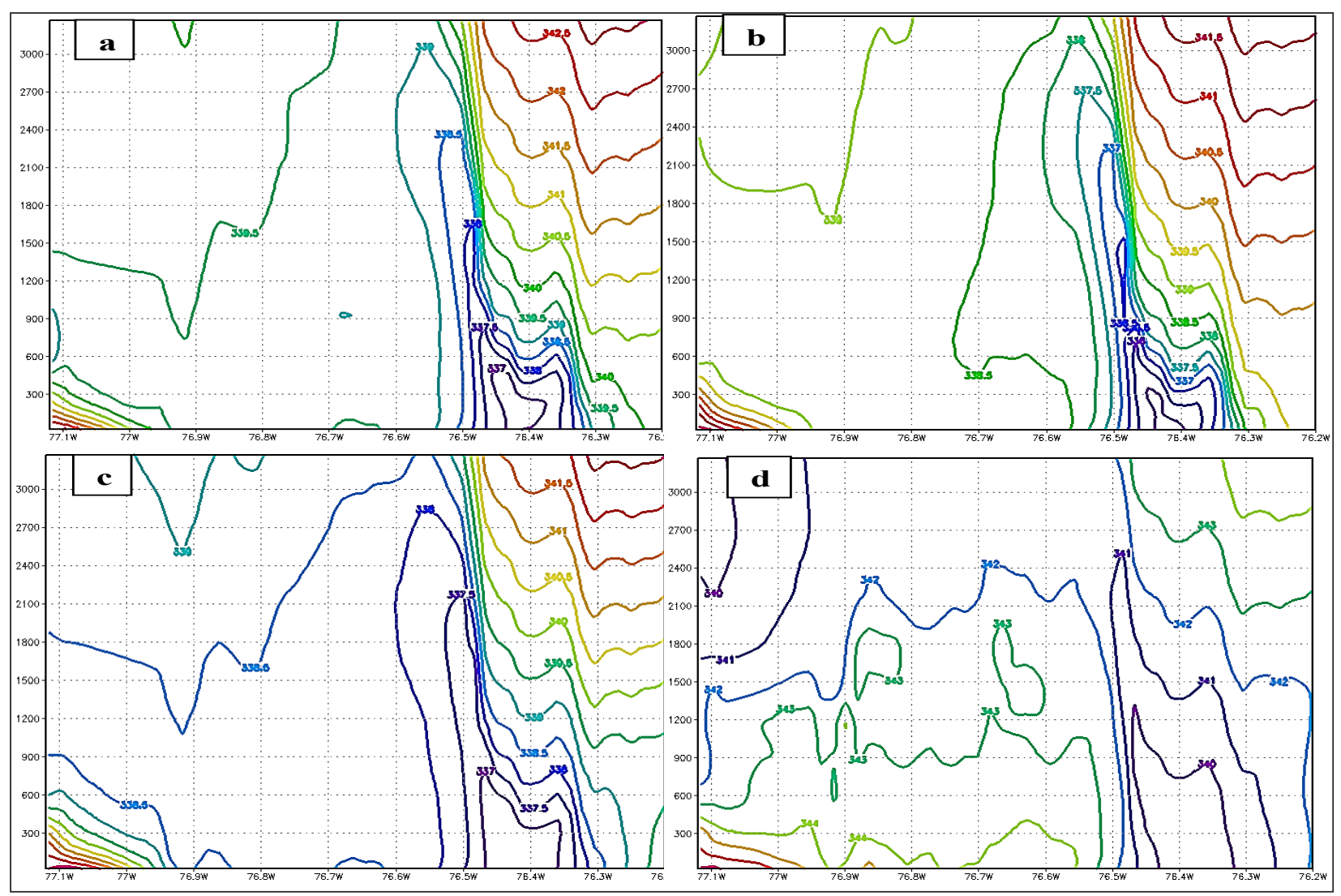

Figura 11. Distribución vertical de temperatura potencial equivalente para el día 23 de marzo para las horas $06 Z$ (a), $12 \mathrm{Z}$ (b), $18 \mathrm{Z}$ (c) y $24 \mathrm{Z}$ (d), respectivamente
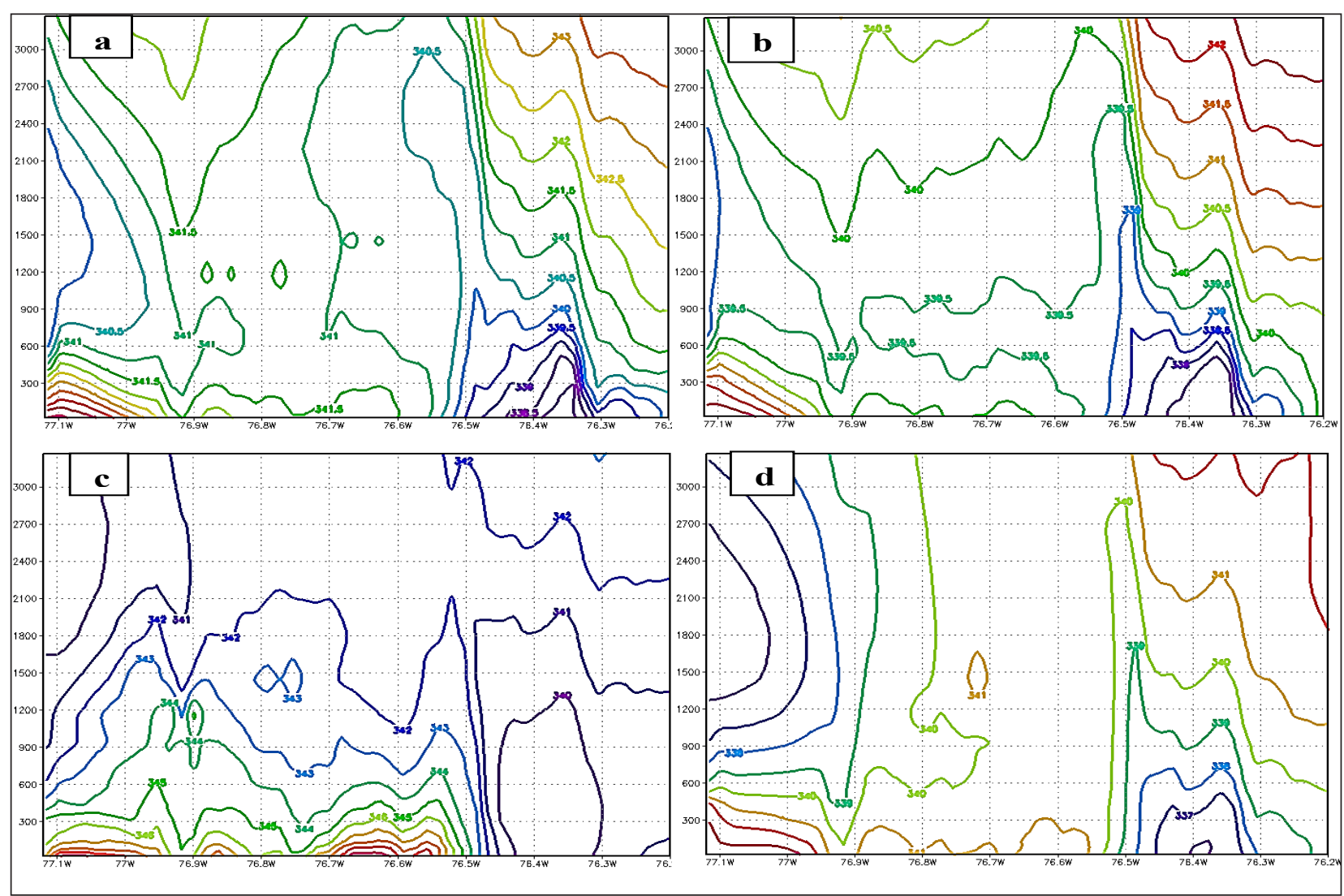

Figura 12. Distribución vertical de temperatura potencial equivalente para el día 24 de marzo para las horas 06Z (a), $12 \mathrm{Z}$ (b), $18 \mathrm{Z}$ (c) y $24 \mathrm{Z}$ (d), respectivamente 


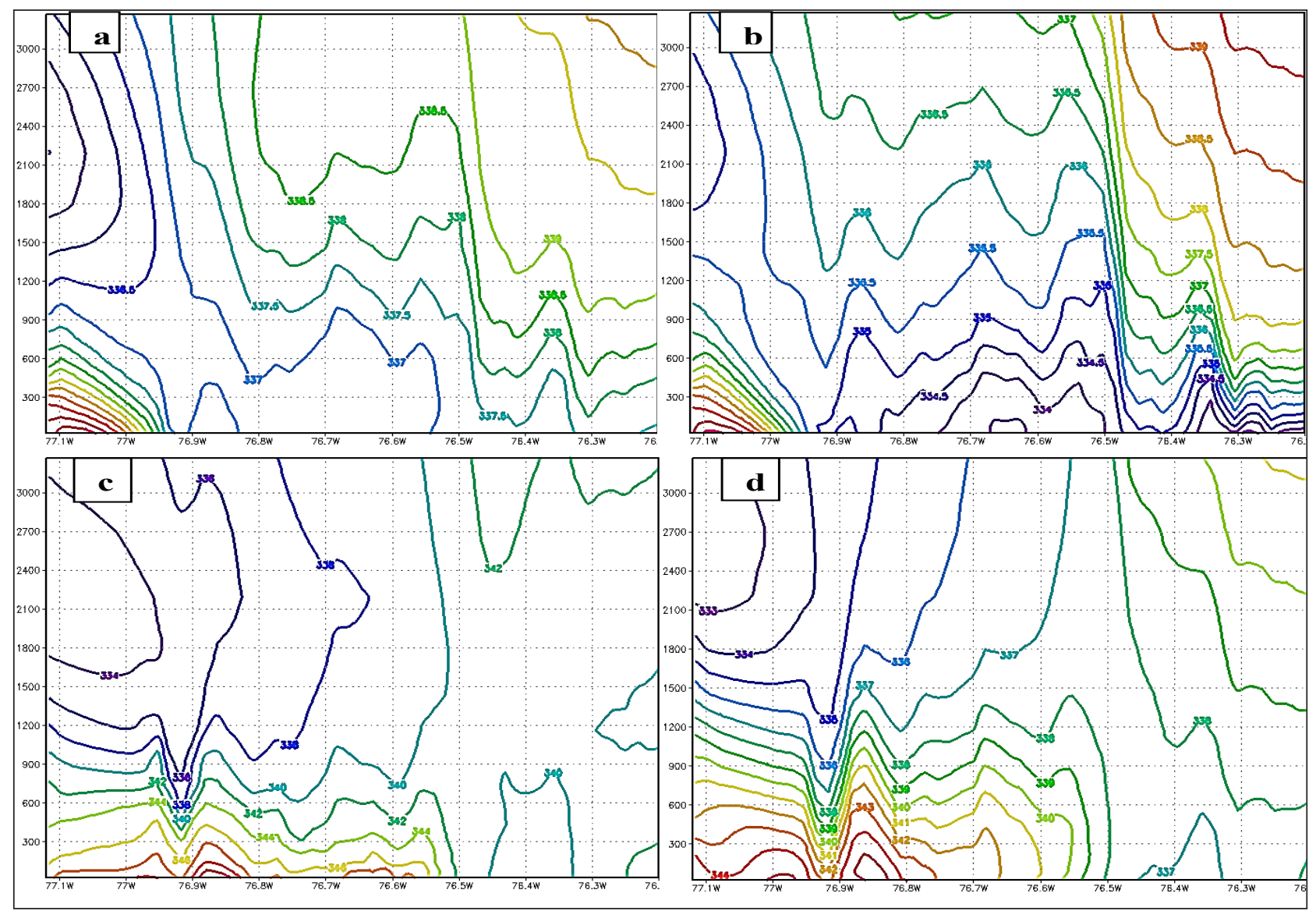

Figura 13. Distribución vertical de temperatura potencial equivalente para el día 25 de marzo para las horas $06 Z$ (a), $12 \mathrm{Z}$ (b), $18 \mathrm{Z}$ (c) y $24 \mathrm{Z}$ (d), respectivamente

anticipación.

Los histogramas de los datos registrados confirmaron la misma tendencia de variación altitudinal de los datos pronosticados para el día 23 de marzo.

Los factores forzantes de las precipitaciones asociadas al huayco ocurrido en Chosica fueron la barrera orográfica que propició convección forzada y levantamiento de la capa atmosférica, atmósfera potencialmente inestable $(\mathrm{d} \theta \mathrm{e} /$ $\mathrm{dz}<0$ ), y la presencia de los flujos del oeste o sistemas del pacífico.

\section{Literatura citada}

BRAMS (Brazilian developments on the Regional Atmospheric Modelling System). 2007. First Time User Guide (en línea). Consultado mayo 2015. Disponible en: http://brams.cptec.inpe.br/docs/bramsguide.

Dillon, E.; García Skabar, Y.; Nicolini, M. 2013. Desempeño del pronóstico de precipitación de modelos de alta resolución, en un área limitada: análisis de la estación de verano: 2010-2011. Meteorologica. 38 (2): 69-89.

García Skabar, Y.; Salio, P.; Nicolini, M. 2012. Verificación de los pronósticos del modelo BRAMS centrado en la región subtropical de Sudamérica. Revista Brasileña de Meteorología, 27 (3): 291- 306.
SENAMHI (Servicio Nacional de Meteorología e Hidrología, PE). 2015. Boletín Climático Nacional (en línea). Consultado ago. 2015. Disponible en: http:// www.senamhi.gob.pe/load/file/02215SENA-22.pdf.

Villacorta, S.; Nuñez, S.; Huarez, C.; Fidel, L. 2015. Evaluación geológica y consecuencias de los huaicos de Chosica del 23-03-15: crónica de un desastre anunciado. INGEMMET (Instituto Geológico Minero y Metalúrgico, PE). 


\section{ANEXO}

Campo de viento en cuatro niveles: $24 \mathrm{~m}$ y $731 \mathrm{~m}$ (a), 2680m y 3266m (b), y topografía: áreas sombreadas en colores,

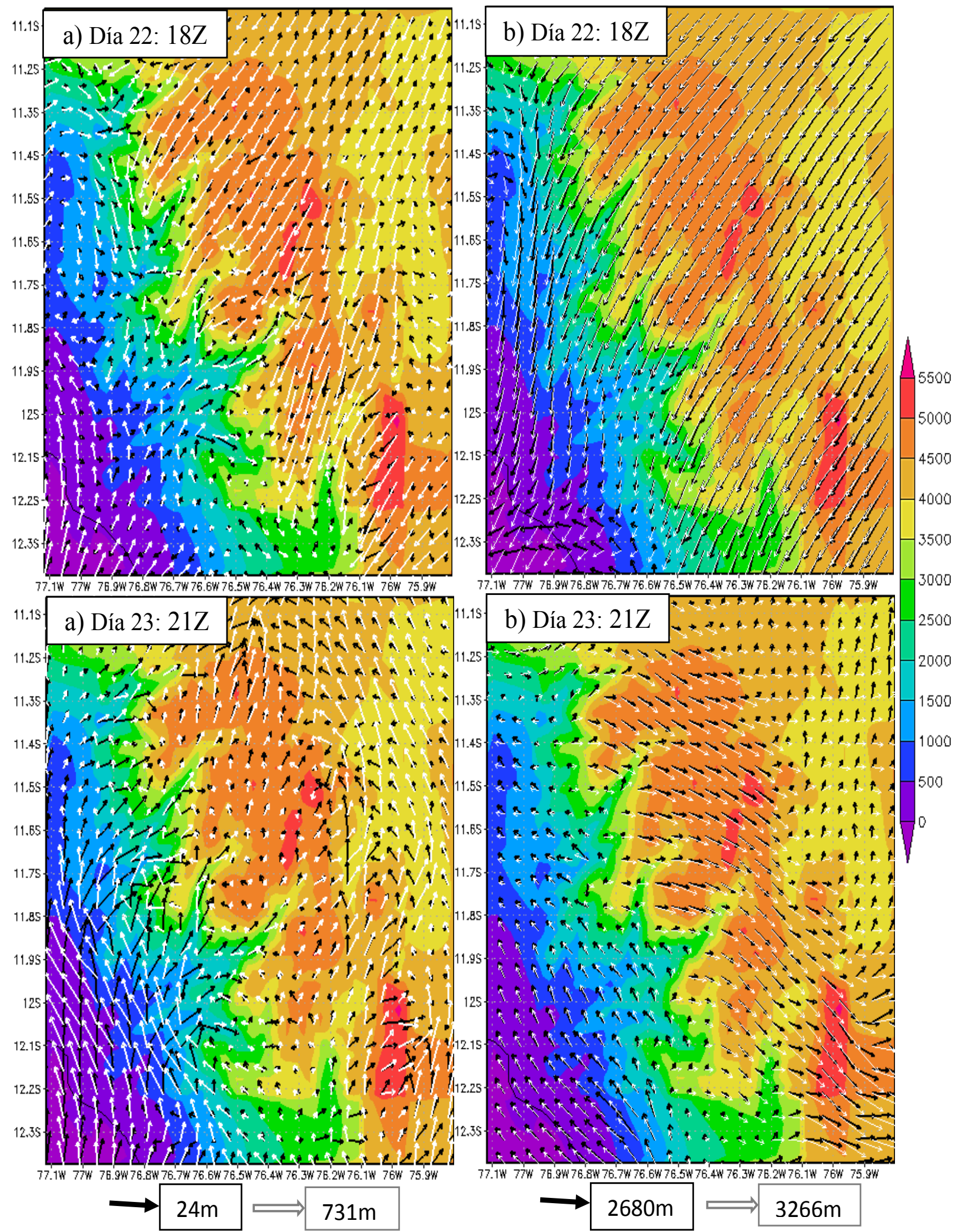




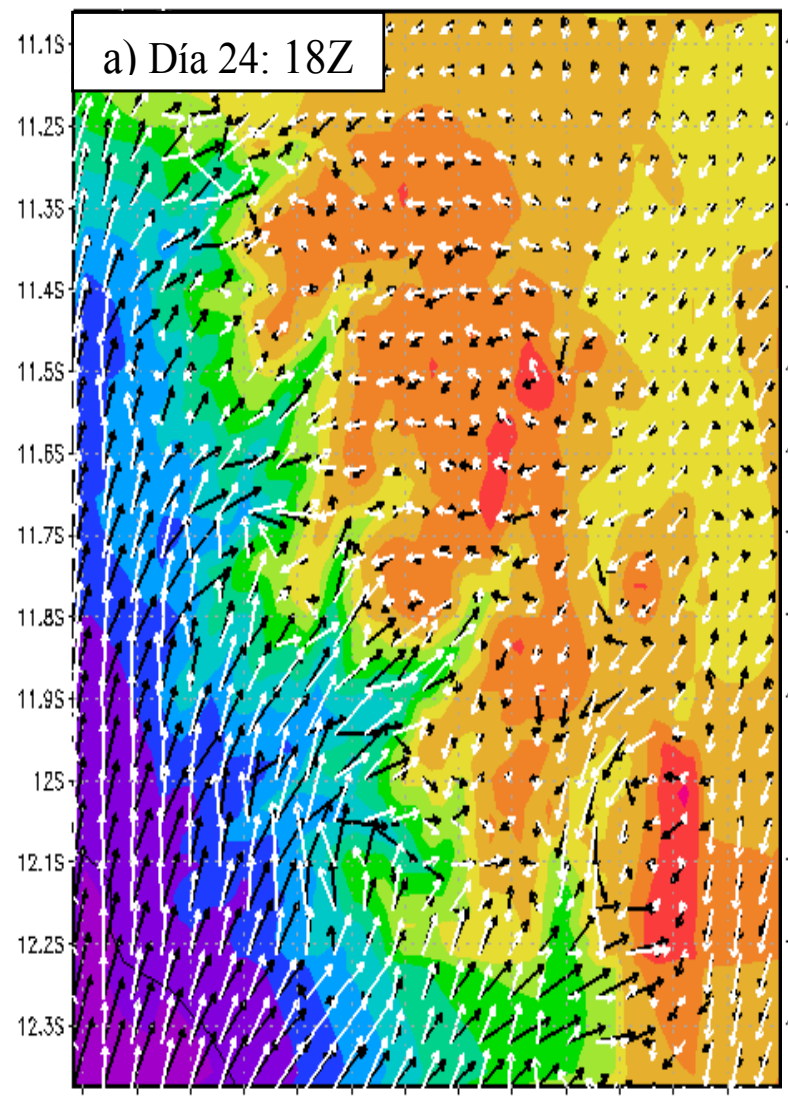

$77.1 \mathrm{~W} 77 \mathrm{~W}$ 76.9W76.8W 76.7W76.6W76.5W76.4W 76.3W76.2W76.1W 76W 75.9W
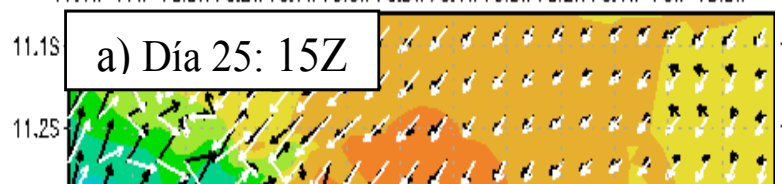

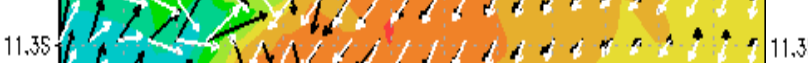

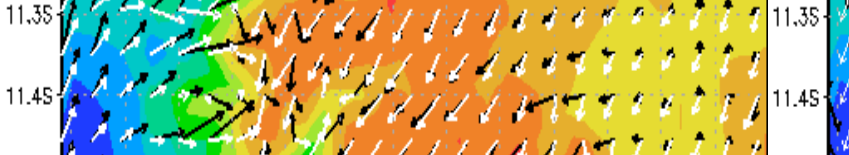

$11.55(+1)+11.55$

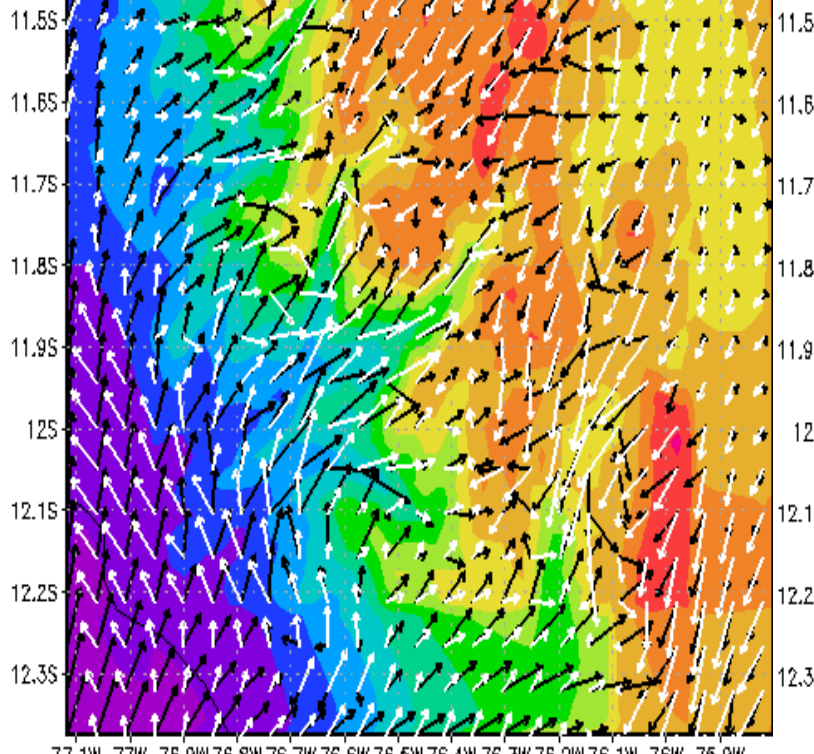

77.1W $77 \mathrm{~W} 76.9 \mathrm{~W} 76.8 \mathrm{~W} 76.7 \mathrm{~W} 76.6 \mathrm{~W} 76.5 \mathrm{~W} 76.4 \mathrm{~W} 76.3 \mathrm{~W} 76.2 \mathrm{~W} 76.1 \mathrm{~W} 76 \mathrm{~W} 75 . \mathrm{WW}$

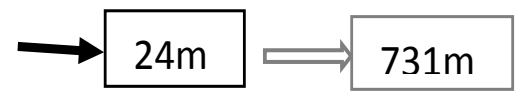

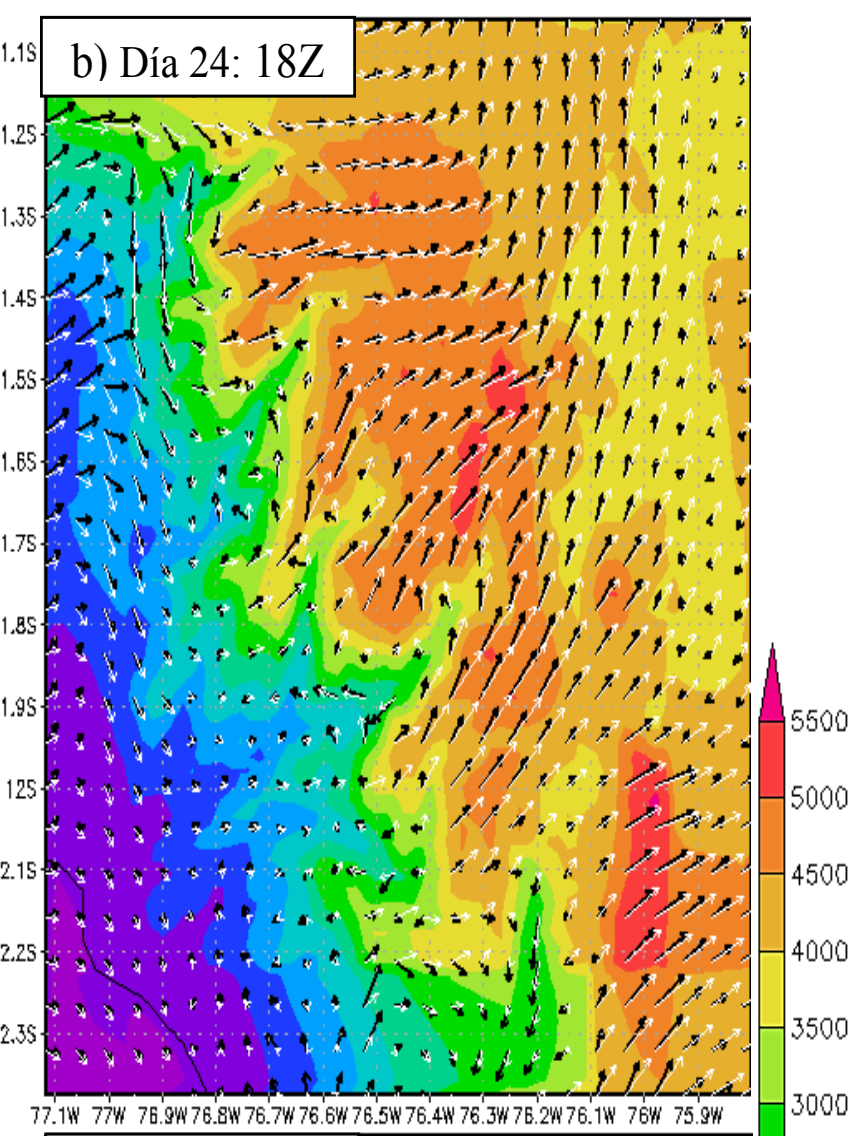

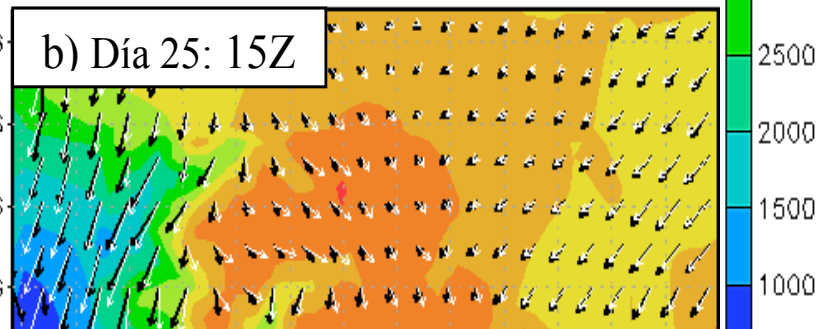

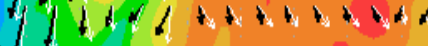
500

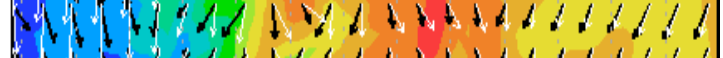

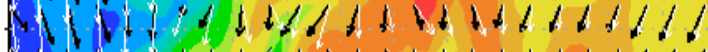
Af $1+1+1+1+1+1+161$

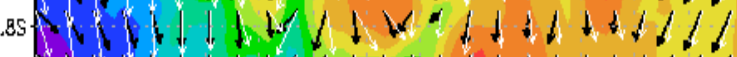

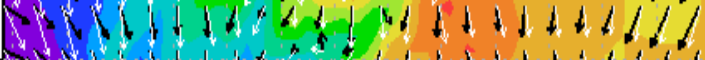

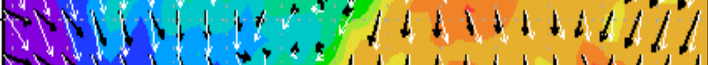
$25+1)+1+4+1+0 / 1$

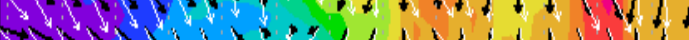
$15+1+1+2+1+1+11$ NDID/ $11 / 11111$ $+121111 \% 1111$

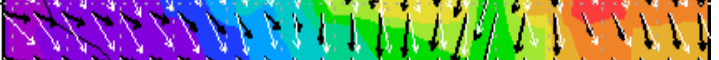

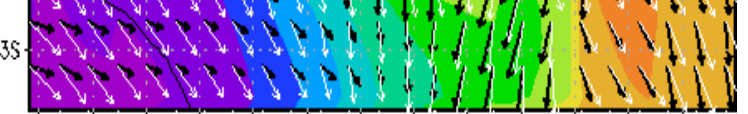

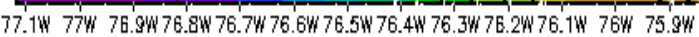

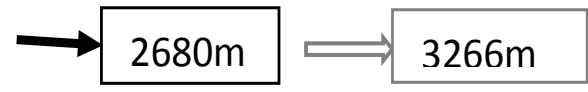

